-Supporting Information-

General Strategy for Enhancing

\title{
Electrochemiluminescence of Semiconductor
}

\section{Nanocrystals by Hydrogen Peroxide and \\ Potassium Persulfate as Dual Coreactants}

\author{
Pan-Pan Dai, Tao Yu, Hai-Wei Shi, Jing-Juan Xu*, Hong-Yuan Chen*
}

State key Laboratory of Analytical Chemistry for Life Science and Collaborative

Innovation Center of Chemistry for Life Sciences, School of Chemistry and Chemical

Engineering, Nanjing University, Nanjing 210023, China

Corresponding authors

*Tel/fax: +86-25-89687294. E-mail: xujj@nju.edu.cn.

*Tel/fax: +86-25-89684862. E-mail: hychen@nju.edu.cn. 


\section{EXPERIMENTAL SECTION}

Reagents. Titanium foils $(99.7 \%$ pure, $0.25 \mathrm{~mm}$ thick) were obtained from Sigma-Aldrich (St. Louis, MO). $\mathrm{H}_{2} \mathrm{O}_{2}$ with analytical grade was purchased from Shanghai Chemical Reagent Company (Shanghai, China). Hydrofluoric acid and potassium peroxydisulfate were purchased from Nanjing Chemical Co. Ltd. 0.1 M phosphate buffer solution $\left(\mathrm{KH}_{2} \mathrm{PO}_{4}-\mathrm{K}_{2} \mathrm{HPO}_{4}-\mathrm{NaCl}\right.$; PBS) containing $0.1 \mathrm{M} \mathrm{K}_{2} \mathrm{~S}_{2} \mathrm{O}_{8}$ and (or) $\mathrm{H}_{2} \mathrm{O}_{2}$ with different concentration was used for ECL detection. All other reagents were of analytical grade and used as received. All aqueous solutions were prepared using ultra-pure water (Milli-Q, Millipore).

Apparatus. The electrochemical and ECL emission measurements were obtained using an MPI-A multifunctional electrochemical and chemiluminescent analytical system (Remax Electronic Instrument Limited Co., Xi'an, China, $350 \mathrm{~nm}-650 \mathrm{~nm}$ ) by cyclic potential scan at room temperature, and the voltage of the PMT was set at in the process of detection. The experiments were carried out with a conventional three-electrode system: $\mathrm{TiO}_{2} \mathrm{NTs}$ substrate or a GCE with $3 \mathrm{~mm}$ diameter modified with CdS NCs film as working electrode, a Pt wire as the counter electrode, an SCE electrode as the reference electrode. ECL spectra were obtained by a series of optical filters $(420,440,460,480,500,520,540,560,580,600,620 \mathrm{~nm})$. Scanning electron microscopy (SEM) images were obtained using an S-3000 N scanning electron microscope (Tokyo, Japan). Electron paramagnetic resonance (EPR) spectra were measured on a Bruker spectrometer (EMX-10/12, Bruker, Germany) with DMPO as a spin-trapping agent at room temperature, and analyzed by WINEPR Sim Fonia software (Bruker, Germany). X-ray diffraction (XRD) was performed with an X-ray 
diffractometer (XRD-6000, Shimadzu, Japan) using a $\mathrm{Cu}$ Ka source $(1=0.154060 \mathrm{~nm})$ at $40 \mathrm{kV}, 30 \mathrm{~mA}$ in the range of $20^{\circ}<2 \theta<80^{\circ}$ at a scan rate of $4.0^{\circ} \mathrm{min}^{-1}$.

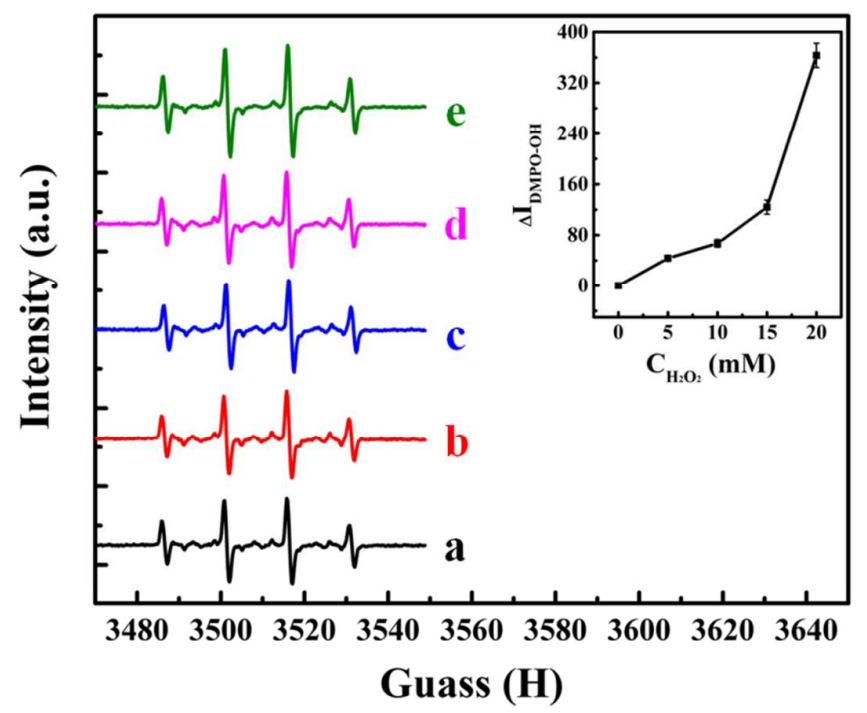

Figure S1. EPR spectra of 0.1 M PBS buffer (pH 7.5) containing 0 (a), 5 (b), 10.0 (c), 15.0 (d) and $20.0 \mathrm{mM} \mathrm{H}_{2} \mathrm{O}_{2}$ (e) with $\mathrm{K}_{2} \mathrm{~S}_{2} \mathrm{O}_{8}$ and DMPO fixed at $0.1 \mathrm{M}$ and $100 \mathrm{mM}$ respectively, were recorded after applying the potential of $-1.6 \mathrm{~V}$ on the $\mathrm{TiO}_{2}$ electrode for $150 \mathrm{~s}$; Inset: effects of $\mathrm{H}_{2} \mathrm{O}_{2}$ concentration on the increment in EPR signal of DMPO-OH emerged at $3501 \mathrm{G}$ before and after addition of $\mathrm{H}_{2} \mathrm{O}_{2}(\Delta \mathrm{I})$. 


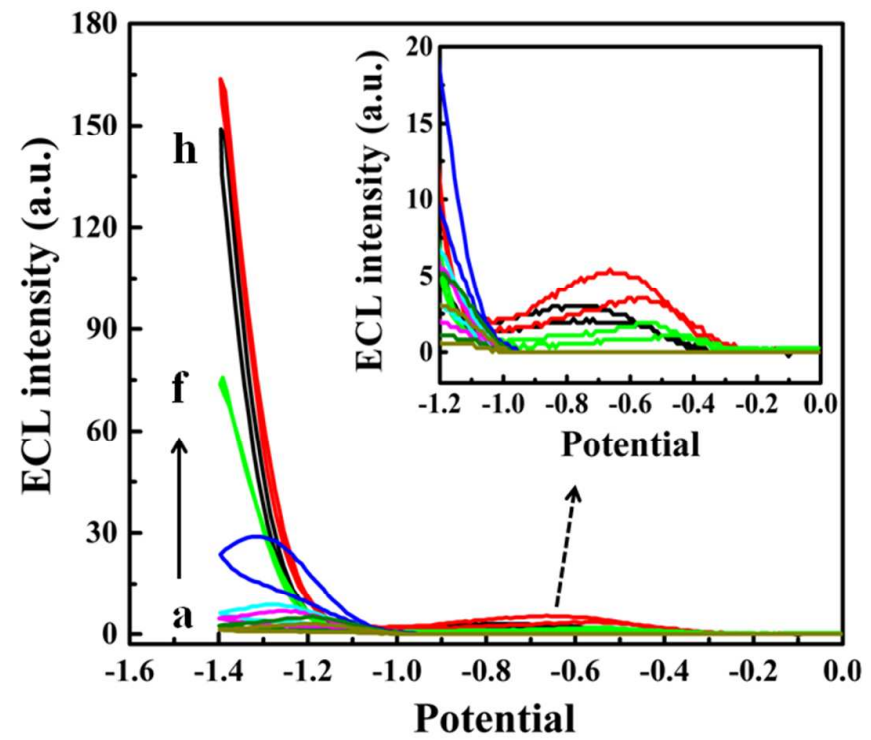

Figure S2. Effects of $\mathrm{H}_{2} \mathrm{O}_{2}$ concentration (a-h: 0, 0.005, 0.01, 0.05, 0.5, 5, 10, 15 $\mathrm{mM}$ ) on the ECL intensity of $\mathrm{TiO}_{2} \mathrm{NTs}$.
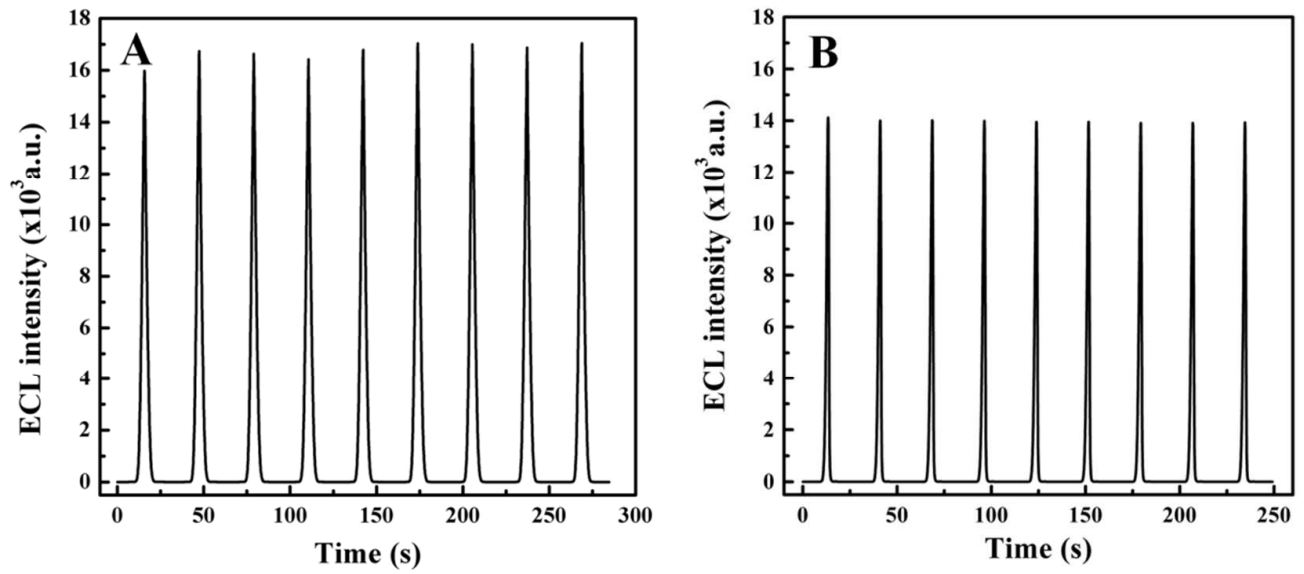

Figure S3. The excellent stability of enhanced ECL of $\mathrm{TiO}_{2} \mathrm{NTs}(\mathrm{A})$ and CdS NCs (B) under the optimum condition. 